\title{
Anti- SOX1 antibody-positive paraneoplastic neurological syndrome presenting with Lambert- Eaton myasthenic syndrome and small cell lung cancer: a case report
}

\section{Wenqi Zheng}

Affiliated Hospital of Inner Mongolia Medical College https://orcid.org/0000-0002-2970-3768

\section{Xiaolei Wang}

Affiliated Hospital of Inner Mongolia Medical College

Lihua Sun

Affiliated Hospital of Inner Mongolia Medical College

Hui Deng

Affiliated Hospital of Inner Mongolia Medical College

Yanqiu Han

Affiliated Hospital of Inner Mongolia Medical College

Chunyang Li ( $\sim$ lichunyang770905@163.com)

\section{Case report}

Keywords: Anti- SOX1antibody, PNS, LEMS, SCLC

Posted Date: April 18th, 2019

DOI: https://doi.org/10.21203/rs.2.9166/v1

License: (c) (i) This work is licensed under a Creative Commons Attribution 4.0 International License. Read Full License 


\section{Abstract}

Background Paraneoplastic neurological syndromes (PNS) are rare disorders affecting any part of the central, peripheral or autonomic nervous system that occur in association with cancer. Among cancer patients, less than $1 \%$ overall develop PNS. Anti-SOX1 antibodies positive paraneoplastic neurological disorders are rare and most often associated with small cell lung cancer (SCLC). Case presentation Herein, we report a case of a 61-year-old male presented with an unusual anti-SOX1 positive PNS. Electrodiagnostic study showed notable low amplitude motor potentials and high amplitude motor potentials of the right tibialis anterior suggesting the presence of Lambert-Eaton myasthenic syndrome (LEMS). Typical MRI and PET-CT found a hyperintense lesion with contrast enhancement in the thorax in front of 5-6 centrum of vertebrae, and thoracoscopic biopsy revealed pathological findings for SCLC. Because the diagnosis was made in time, the patient is currently receiving chemotherapy and radiotherapy for the cancer at Chinese PLA General Hospital, and the clinical symptoms improved obviously. Conclusions The comprehensive screening of onconeural antibodies in PNS-suspicious cases combined with early diagnosis and treatment of tumor are important for achieving a good outcome.

\section{Background}

Paraneoplastic neurological syndrome (PNS) are rare immune-mediated neurological disorders that is caused by the remote immune cross-reaction between antigens expressed by neurons and tumor cells[1]. It can effects of the underlying neoplasm and may affect central, peripheral or autonomic nervous system and accounts for a constellation of clinical features[2]. PNS of the central nervous system can appear as limbic encephalitis, paraneoplastic cerebellar degeneration (PCD), or opsoclonus-myoclonus syndrome (OMS), whereas PNS of the peripheral nervous system can present as neuropathy, disorders of the neuromuscular transmission like Lambert-Eaton myasthenic syndrome (LEMS) or Myasthenia gravis (MG), et al [3, 4]. Among cancer patients, less than $1 \%$ overall develop PNS. The most prominently associated tumor is small cell lung cancer (SCLC), up to 3-5\% of PNS occurs with SCLC[5] followed by other tumors such as lymphoid, breast, thymic and ovarian[6, 7].

In the last years, the detection of defined anti-neuronal auto antibodies has improved the diagnosis of PNS. Many of well-characterized anti-neuronal antibodies have been described to associate with PNS include the anti-Hu, anti-Yo antibody, or other increasing number of anti-neuronal autoantibodies (Ma1, Ma2/Ta, CV2/CRMP5, and amphiphysin) [3, 8]. As an anti-glial antibody associated with PNS, anti-SOX1 antibodies target a protein of the Sry-like high mobility group superfamily of developmental transcription factors that are preferentially expressed in the nuclei of Bergmann glia in the adult cerebellum $[9,10]$. They were initially found in the sera of patients with SCLC without neurological disorders[11] but were absent in patients with other tumors [12]. In addition, anti-SOX1 antibodies were found in majority of patients with voltage-gated calcium channel (VGCC) antibody-associated LEMS predicting the presence of SCLC, except for the patients with a non-paraneoplastic LEMS [9]. 
We report the case of a patient with neurological symptoms, electro-diagnostic studies, laboratory tests and pathology examination consistent with PNS presenting with anti-SOX1 antibody positive LEMS, in which a SCLC was subsequently diagnosed. He later developed generalized seizure and progressive confusion suggestive of limbic encephalitis (LE). This case demonstrates the importance of a multidisciplinary team approach in regard to the early recognition of PNS, and the importance of screening for additional autoantibodies in the presence of atypical symptoms. Moreover, when the neurological syndromes and paraneoplastic neuronal autoantibodies are present, aggressive search and rule out underlying cancer is crucial.

\section{Case Presentations}

The patient was a 61 year-old Chinese male, with a perennial drinking history and no history of smoking, presented with a self-reported three month bitter taste in the mouth causing some discomfort and unpleasantness during meals but no obvious dysphagia. In the following months, his gait gradually became unsteady and developed weakness of lower limbs. He had no symptom fluctuation over the course of the day but the skeletal muscle fatigue was increasing. His neurologic examination revealed proximal muscles of lower limb weakness at grade 4 on the Medical Research Council scale, poor deep tendon reflexes and heel-knee-shin test, and exhibited signs associated with acute cerebellar degeneration, such as dizziness, mild dysarthria, vertigo and clear ataxia. He displayed saccadic eye movements and nystagmus, symptoms of diplopia and barrier to sensory plane developed over the next few days. His cognitive functions and cranial nerves were normal. He began an evaluation that included laboratory study notable for positive antinuclear antibody (ANA) at 1:80 (reference $<1: 40$ ), elevated serum ferritin (SF) at $509.6 \mathrm{ng} / \mathrm{ml}$ (reference $80-130 \mathrm{ng} / \mathrm{ml}$ ), positive treponema pallidum antibody (TP) at 9.85 (reference $0-1$ ). However, venereal disease research laboratory test (VDRL) showed reagin of cerebrospinal fluid (CSF) was negative. A CSF examination showed positive Pandy's test, an elevated level of total protein $0.824 \mathrm{~g} / \mathrm{L}$ (reference $0.15-0.45$ ), and an elevated IgG level of $72.1 \mathrm{mg} / \mathrm{L}$ (reference 6.333.3). The number of mononuclear cells was negative. In addition, CSF cytology revealed no atypical cells. At that time, blood count, anti-neutrophil cytoplasmic antibodies (ANCA), biochemical all items, erythrocyte sedimentation rate (ESR), renal and liver function, coagulation and serum electrolytes were within normal range.

Myasthenia gravis serology testing showed that anti-striated muscle antibodies and anti-myocardium antibody were negative, and anti-acetylcholine receptor (AchR) binding antibody at $0.01 \mathrm{nmol} / \mathrm{L}$ (reference negative $₫ 40 \mathrm{nmol} / \mathrm{L}$; equivocal $40 \mathrm{nmol} / \mathrm{L}-50 \mathrm{nmol} / \mathrm{L}$; 『positive $50 \mathrm{nmol} / \mathrm{L}$ ), however the antiSOX1 antibody was positive, confirming the presence of LEMS. Electro diagnostic study showed notable low amplitude motor potentials of the right tibialis anterior and left trapezius, a decrement with low frequency $(3 \mathrm{~Hz})$ repetitive nerve stimulation (RNS). Meanwhile, the right tibialis anterior was tested with high-frequency $(50 \mathrm{~Hz})$ RNS, where $386 \%$ facilitation was observed, consistent with LEMS (Fig. 1).

The patient was to begin investigational treatment with pyridostigmine bromide tablets for a week (60mg, Q8h, P.0), symptoms of diplopia disappeared but he still felt weak. Soon afterwards, he developed several 
days of progressive confusion and psychiatric symptoms suggestive of LE. Although the well characterized onconeural antibodies including Hu-Ab, Ri-Ab, Tr-Ab, GAD-Ab, Yo-Ab, CV2-Ab, ANNA-3-Ab, PCA2-Ab, Ma2-Ab, and anti Amphiphysin-Ab were all tested negative, but since the magnetic resonance imaging (MRI) (Fig.2) and PET-CT(Fig.3A, B) demonstrated hyper intense lesions with contrast enhancement of thorax in front of 5-6 centrum, therefore anti-SOX1 antibody-positive PNS associated with SCLC was suspected. A subsequent thoracoscopic biopsy of the lung tissue was performed at Chinese PLA General Hospital, this revealed pathological findings for SCLC in mediastinal lymph nodes. The patient is currently receiving chemotherapy and radiotherapy for the cancer at Chinese PLA General Hospital.

\section{Discussion And Conclusions}

We present a case of anti-SOX1 associated PNS presenting with LEMS and LE in a patient with SCLC tumor. Our case highlights several characteristic features of anti-SOX1 associated PNS, including 'classical' paraneoplastic neurological syndromes, with well-characterized onconeural antibodies and pathologically determined cancer. PNS occur with increased frequency in patients with cancer, they are often therapy-refractive, relentlessly progressive syndromes. In the clinical features of 'classical' PNS, the most relevant advance is LEMS. LEMS occurs as a paraneoplastic neurological syndrome in about 50$60 \%$ of the cases $[3,13]$. As a rare autoimmune disorder of neuromuscular junction transmission, LEMS often presents with clinical features of proximal muscle weakness, diminished deep tendon reflexes, and autonomic dysfunction, and the associated tumor is almost solely SCLC [14]. As an aggressive, neuroectodermal origin tumor, SCLC is most strongly associated with history of smoking. Although SCLC initially responsive to chemotherapy, the tumor is often fatal, with a median survival from diagnosis of only 8 to 10 months, as in our patient. More rarely, as in the case of our patient with SCLC who is a nonsmoker, with no symptoms of weight loss. Although the patient had a long history of heavy drinking, but he had been sober for almost a year. The SCLC induction reason of this patient remains unclear.

In most of the cases, the manifestations of PNS occur before the diagnosis of pulmonary carcinoma [8], and almost always antedate its diagnosis. Furthermore, given the fact that occurrence of PNSs are rare, many cases are not readily diagnosed as PNS. The identification of anti-neuronal antibodies is crucial. Even if several associated antibodies have been reported [8], less than $50 \%$ of patients with PNS harbor paraneoplastic antibodies [3]. Anti-SOX1 antibodies, also known as anti-glial nuclear antibody (AGNA), are specifically for paraneoplastic neurological disorders. Since SOX1 is expressed in neuronal precursor cells in the developing central nervous system, it has been used as an early marker of neural stem cells [15]. While anti- SOX1 antibodies are most often associated with SCLC [16]. In a recent study, reactivity with SOX 1 antibodies were found in $64 \%$ of patients with LEMS and SCLC, in $32 \%$ of patients with PNS and SCLC, and in $22 \%$ of patients with SCLC alone $[9,17]$. In our case, routine antibodies to PNS such as $\mathrm{Hu}-\mathrm{Ab}$, Ri-Ab, Tr-Ab, GAD-Ab, Yo-Ab were tested negative, but SOX1 was found to be strongly positive, this finding encourages the testing of comprehensive onconeural antibodies including SOX1 in PNSsuspicious cases. Meanwhile, when neurologists evaluated a patient with LEMS, an underlying tumor 
should be routinely screened. The initial and most importantly the early treatment of the tumor perhaps will lead to an improved prognosis.

\section{Abbreviations}

PNS: Paraneoplastic neurological syndromes; SCLC: small cell lung cancer; LEMS: Lambert-Eaton myasthenic syndrome; PCD: paraneoplastic cerebellar degeneration; OMS: opsoclonus-myoclonus syndrome; MG: Myasthenia gravis; VGCC: voltage-gated calcium channel; LE: limbic encephalitis; SF: serum ferritin; TP: treponema pallidum antibody; VDRL: venereal disease research laboratory test; CSF: cerebrospinal fluid; ANCA: anti-neutrophil cytoplasmic antibodies; ESR: erythrocyte sedimentation rate; AchR: anti-acetylcholine receptor; RNS: repetitive nerve stimulation; MRI: magnetic resonance imaging; AGNA: anti-glial nuclear antibody.

\section{Declarations}

\section{Ethics approval and consent to participate}

The patient with anti-SOX1 antibody-positive PNS presenting with LEMS and small cell lung cancer was admitted to the Affiliated Hospital of Inner Mongolia Medical University between March and May 2018. This study was authorized by the ethical committees of the Affiliated Hospital of Inner Mongolia Medical University. Written informed consent was obtained from legal surrogates of the patient for using the clinical data.

\section{Consent for publication}

Written informed consent was obtained from the patient after treatment for publication of this case report and any accompanying images. A copy of the written consent is available for review by the Editor of this journal.

\section{Availability of data and materials}

The datasets supporting the conclusions of this case report are included within the article.

\section{Competing interests}

The authors declare that they have no competing interests.

\section{Funding}

This study was supported by the National Natural Science Foundation of China 81760367 and 81360213, and the Affiliated Hospital of Inner Mongolia Medical University's Doctor Program (NYFYBS2018). The funders had no role in the design of the study, interpretation of data or in writing the manuscript. They had contributed to the data collection. 
Authors' contributions

CYL and WQZ participated in the design of the case-report and helped to draft the manuscript. HD and YQH evaluated, examined the patient. XLW and LHS performed and interpreted the MRI studies. All authors read and approved the final manuscript.

\section{Acknowledgements}

Not applicable.

\section{References}

1. Voltz R. Paraneoplastic neurological syndromes: an update on diagnosis, pathogenesis, and therapy. Lancet Neurol. 2002; 1(5):294-305.

2. Viaccoz A, Honnorat J. Paraneoplastic neurological syndromes: general treatment overview. Curr Treat Options Neurol. 2013; 15(2):150-168.

3. Graus F, Delattre JY, Antoine JC, Dalmau J, Giometto B, Grisold W, et al. Recommended diagnostic criteria for paraneoplastic neurological syndromes. J Neurol Neurosurg Psychiatry. 2004; 75(8):11351140 .

4. Dalmau JO, Posner JB. Paraneoplastic syndromes affecting the nervous system. Semin Oncol. 1997; 24(3):318-328.

5. Kanaji N, Watanabe N, Kita N, Bandoh S, Tadokoro A, Ishii T, et al. Paraneoplastic syndromes associated with lung cancer. World J Clin Oncol. 2014; 5(3):197-223.

6. Lorusso L, Hart IK, Ferrari D, Ngonga GK, Gasparetto C, Ricevuti G. Autonomic paraneoplastic neurological syndromes. Autoimmun Rev. 2007; 6(3):162-168.

7. Rees JH, Hain SF, Johnson MR, Hughes RA, Costa DC, et al. The role of [18F]fluoro-2-deoxyglucose-PET scanning in the diagnosis of paraneoplastic neurological disorders. Brain. 2001; 124(Pt 11):2223-2231.

8. Darnell RB, Posner JB. Paraneoplastic syndromes involving the nervous system. N Eng/ J Med. 2003; 349(16):1543-1554.

9. Sabater L, Titulaer M, Saiz A, Verschuuren J, Gure AO, Graus F. SOX1 antibodies are markers of paraneoplastic Lambert-Eaton myasthenic syndrome. Neurology. 2008; 70(12):924-928.

10. Sottile V, Li M, Scotting PJ. Stem cell marker expression in the Bergmann glia population of the adult mouse brain. Brain Res. 2006; 1099(1):8-17.

11. Gure AO, Stockert E, Scanlan MJ, Keresztes RS, Jager D, Altorki NK, et al. Serological identification of embryonic neural proteins as highly immunogenic tumor antigens in small cell lung cancer. Proc Natl 
Acad Sci U S A. 2000; 97(8):4198-4203.

12. Graus F, Vincent A, Pozo-Rosich P, Sabater L, Saiz A, Lang B, et al. Anti-glial nuclear antibody: marker of lung cancer-related paraneoplastic neurological syndromes. J Neuroimmunol. 2005; 165(1-2):166-171.

13. Titulaer MJ, Lang B, Verschuuren JJ. Lambert-Eaton myasthenic syndrome: from clinical characteristics to therapeutic strategies. Lancet Neurol. 2011; 10(12):1098-1107.

14. Elrington GM, Murray NM, Spiro SG, Newsom-Davis J. Neurological paraneoplastic syndromes in patients with small cell lung cancer. A prospective survey of 150 patients. $J$ Neurol Neurosurg Psychiatry. 1991; 54(9):764-767.

15. Wegner M. From head to toes: the multiple facets of Sox proteins. Nucleic Acids Res. 1999; 27(6):1409-1420.

16. Graus F, Saiz A, Dalmau J. Antibodies and neuronal autoimmune disorders of the CNS. J Neurol. 2010; 257(4):509-517.

17. Vural B, Chen LC, Saip P, Chen YT, Ustuner Z, Gonen M, et al. Frequency of SOX Group B $($ SOX1, 2, 3) and ZIC2 antibodies in Turkish patients with small cell lung carcinoma and their correlation with clinical parameters. Cancer. 2005; 103(12):2575-2583.

\section{Figures}



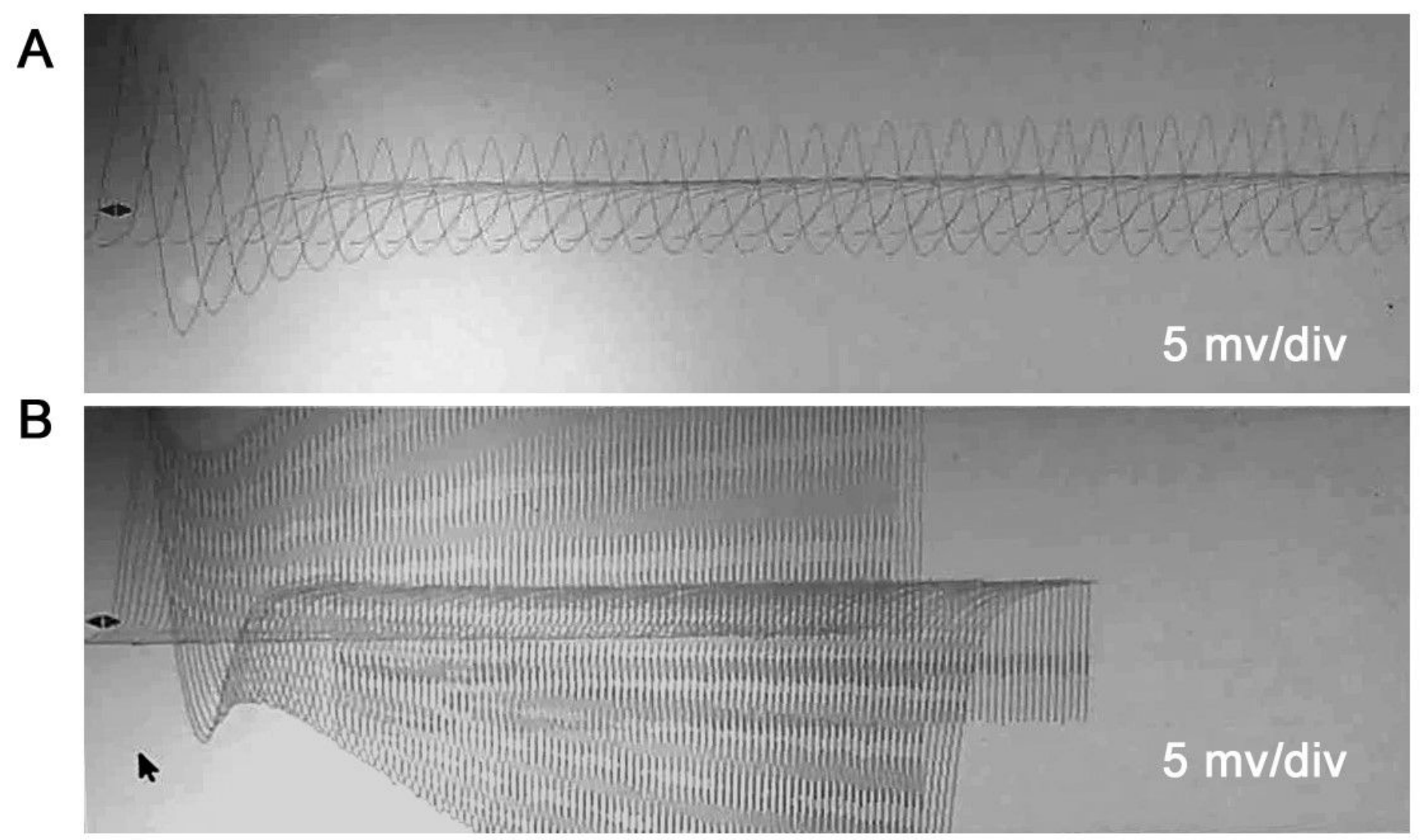

\section{Figure 1}

(A) Electromyography stimulation at $3 \mathrm{~Hz}$ demonstrating a $55.9 \%$ decrement. Gain is $3 \mathrm{mV}$ per division and a sweep speed of 5 milliseconds. (B) An incremental response at high-rate stimulation $(50 \mathrm{~Hz})$ for amplitude of $386 \%$ facilitation in area under curve. 

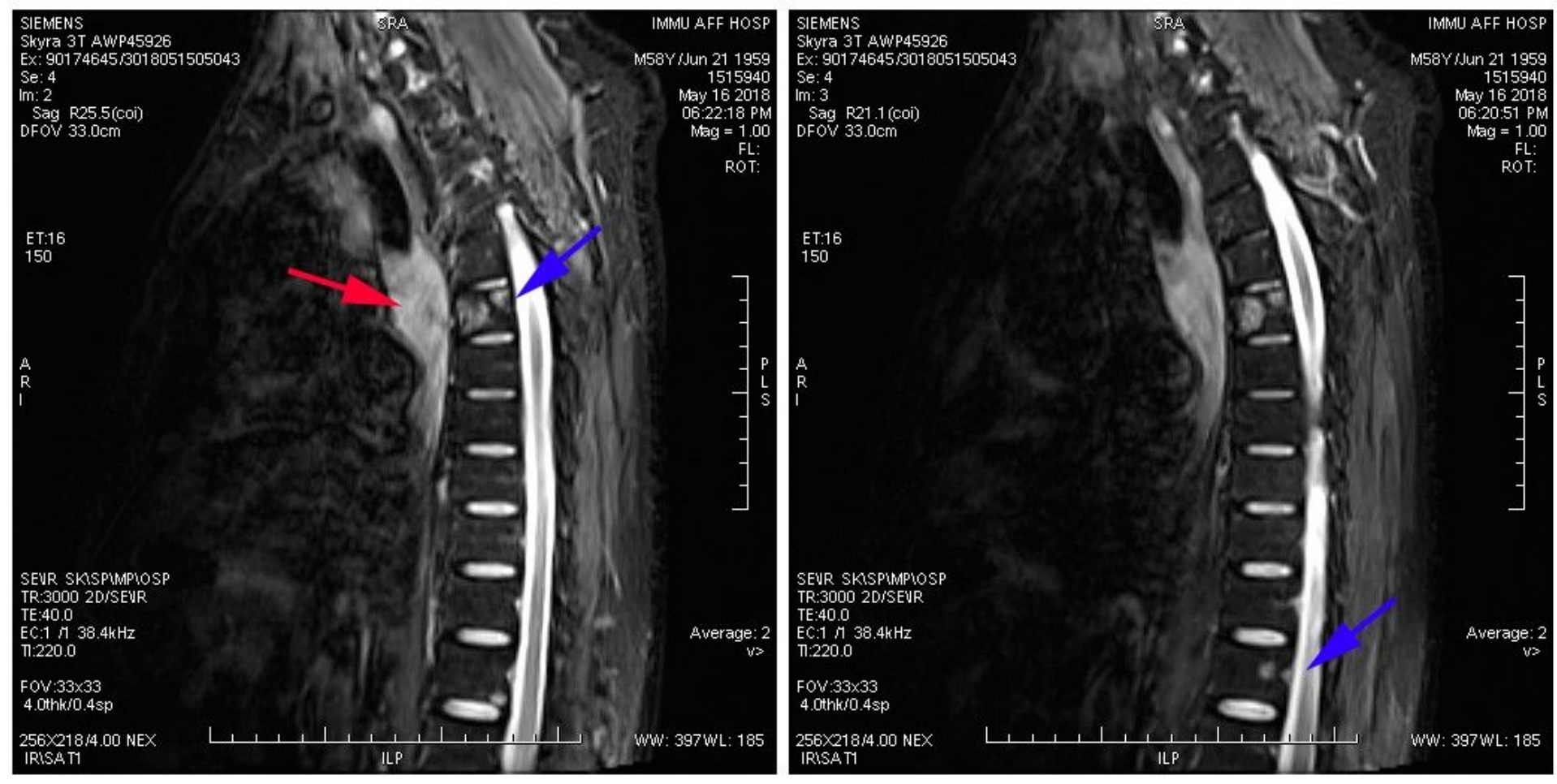

\section{Figure 2}

Spinal MRI demonstrating remarkable enhancement of the thorax in front of 5-6 centrum (as indicated by the red arrow), and revealed to have T6, and T12 destructive vertebral body lesions (as indicated by the blue arrows). 

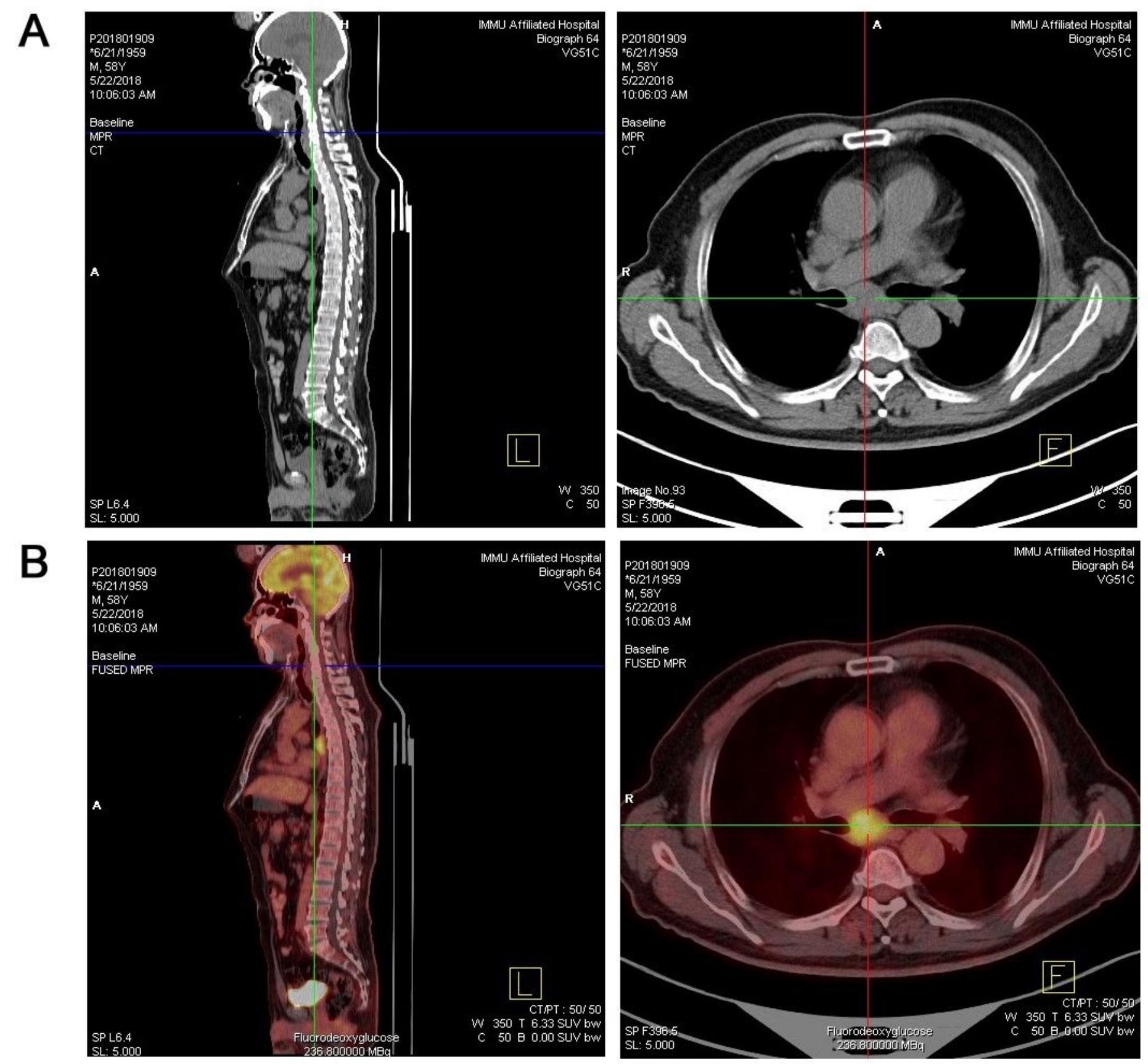

\section{Figure 3}

(A) PET-CT showed multiple enlarged lymph node shadows associated with increased metabolism were found in anterior tracheal vena cava, right pulmonary hilum and subcarinal. (B) [(18)F] fluoro-2-deoxy-Dglucose positron emission tomography/computed tomography imaging revealed area of abnormal FDG accumulation in mediastinal.

\section{Supplementary Files}

This is a list of supplementary files associated with this preprint. Click to download.

- CAREchecklistEnglish20131.pdf 